\title{
BMJ Open Targeting strategies of mHealth interventions for maternal health in low and middle-income countries: a systematic review protocol
}

\author{
Onaedo llozumba, ${ }^{1,2,3}$ Ibukun-Oluwa Omolade Abejirinde, ${ }^{1,2,3}$ Marjolein Dieleman, ${ }^{1}$
} Azucena Bardaji, ${ }^{3}$ Jacqueline E W Broerse, ${ }^{1}$ Sara Van Belle ${ }^{2}$

To cite: llozumba 0, Abejirinde I-00, Dieleman M, et al. Targeting strategies of mHealth interventions for maternal health in low and middle-income countries: a systematic review protocol. BMJ Open 2018;8:e019345. doi:10.1136/ bmjopen-2017-019345

- Prepublication history and additional material for this paper are available online. To view these files, please visit the journal online (http://dx.doi. org/10.1136/bmjopen-2017019345).

Received 28 August 2017 Revised 16 January 2018 Accepted 22 January 2018

Check for updates

${ }^{1}$ Faculty of Sciences, Vrije universiteit Amsterdam, Amsterdam, The Netherlands ${ }^{2}$ Department of Public Health, Instituut voor Tropische Geneeskunde, Antwerpen, Belgium

${ }^{3}$ ISGlobal, Barcelona Ctr. Int. Health Res. (CRESIB), Hospital Clínic - Universitat de Barcelona, Barcelona, Spain

Correspondence to

Onaedo llozumba;

ona.ilozumba@vu.nl

\section{ABSTRACT}

Introduction Recently, there has been a steady increase in mobile health (mHealth) interventions aimed at improving maternal health of women in lowincome and middle-income countries. While there is evidence indicating that these interventions contribute to improvements in maternal health outcomes, other studies indicate inconclusive results. This uncertainty has raised additional questions, one of which pertains to the role of targeting strategies in implementing mHealth interventions and the focus on pregnant women and health workers as target groups. This review aims to assess who is targeted in different mHealth interventions and the importance of targeting strategies in maternal mHealth interventions. Methods and analysis We will search for peer-reviewed, English-language literature published between 1999 and July 2017 in PubMed, Web of Knowledge (Science Direct, EMBASE) and Cochrane Central Registers of Controlled Trials. The study scope is defined by the Population, Intervention, Comparison and Outcomes framework: $\mathrm{P}$, community members with maternal or reproductive needs; I, electronic health or mHealth programmes geared at improving maternal or reproductive health; C, other non-electronic health or mHealth-based interventions; 0 , maternal health measures including family planning, antenatal care attendance, health facility delivery and postnatal care attendance.

Ethics and dissemination This study is a review of already published or publicly available data and needs no ethical approval. Review results will be published in a peer-reviewed journal and presented at international conferences.

PROSPERO registration number CRD42017072280.

\section{INTRODUCTION}

Mobile health (mHealth) involves the use of mobile phones or portable devices such as personal digital assistants (PDAs) for healthcare service delivery. These interventions are usually in the form of direct phone calls, short message service (SMS) messages, voice calls or mobile applications. ${ }^{1}$ mHealth interventions have increasingly been used in improving maternal health outcomes.

\section{Strengths and limitations of this study}

- The protocol adheres to the Preferred Reporting Items for Systematic Reviews and Meta-AnalysesProtocols guidelines for reporting a systematic review protocol.

- The protocol outlines a review process that will involve the use of a systematic literature review, an interdisciplinary team and a narrative synthesis methodology, allowing for an iterative review process.

- The proposed inclusion criteria include quantitative and qualitative studies and a narrative synthesis methodology. This combination presents an opportunity for the review to answer questions not only about 'what' "but also about 'why and how'.

- The protocol proposes the utilisation of multiple tools to assess the strength of evidence including the Downs and Black (1998) checklist for quantitative healthcare studies and the Grading of Recommendations Assessment, Development and Evaluation system.

- Narrative synthesis as a form of content methodology has been criticised for its potential to be biased, and its transparency has been challenged. We address these by using a systematic four-step approach to this synthesis.

The range of available mHealth interventions resulted in Labrique et al developing a framework which identified the 12 common uses of mHealth for maternal and child health, the most predominant being its use for client education and behaviour-change communication. ${ }^{2}$ These interventions may target the supply end of care delivery, aimed at health workers at the facility level, or individual and community levels, that is, the demand-side influencers such as household decision-makers. In this review, we will focus on the individual-level interventions which have focused on strategies shown to work for maternal health including increased 
delivery assistance by a skilled birth attendant, attendance of four or more antenatal care (ANC) visits, and an increased prevalence of contraceptive usage among reproductive-aged women. ${ }^{3}$

mHealth studies which have focused on maternal interventions have yielded conflicting results. There is some positive evidence for the effect on mHealth interventions on outcomes such as ANC attendance and skilled birth attendance at birth and utilisation of oral contraception. ${ }^{4-7}$ However, other studies have reported no statistically significant improvement in reproductive health outcomes following the introduction of mHealth interventions. ${ }^{8-11}$ With the interest in mHealth interventions, there have been a number of reviews on the use of mHealth in low-income and middle-income countries (LMIC). However, they tend to focus specifically on the reproductive health outcomes or the efficacy of mHealth interventions, ${ }^{12-14}$ the use of mHealth by health workers ${ }^{15-17}$ or the acceptance, utilisation and evaluation of these interventions. ${ }^{18-20}$ None of these reviews have focused on the targeting strategies of reproductive mHealth interventions, that is, who are the intervention targets and to what extent this influences observed outcomes.

Literature on maternal health provides strong evidence for the targeting of individuals other than pregnant women. For example, there is evidence that in many contexts husbands play significant and important roles in reproductive health decision-making. ${ }^{21}{ }^{22}$ This variation in decision-makers is rarely addressed and sometimes strategically excluded from the review, as in Lee $e t a l .{ }^{12}$ In addition, other factors that influence reproductive health decision-making and healthcare-seeking behaviour such as literacy, socioeconomic status or access to care are discussed as secondary findings. As a result, there is as yet not much of an understanding regarding whom mHealth interventions are to be designed for, and the relation between targeting strategies in mHealth interventions and reproductive health outcomes.

\section{OBJECTIVE AND RESEARCH QUESTIONS}

The aim of this review is to understand the effects of targeting strategies applied in mHealth interventions for maternal health in LMIC. To address this aim, the following research questions were developed:

1. Who in the community (ie, non-health professionals) a targeted in maternal mHealth interventions?

2. Do targeting strategies differ across LMIC contexts, such as by programme type, funder and so on?

3. What are the sociodemographic characteristics of those targeted in the described mHealth interventions, and what is the nature of mHealth interventions used for these groups?

4. What are the reported intervention outcomes based on characteristics of targeted participants and how are these differences explained?

\section{METHODS AND ANALYSIS}

\section{Protocol registration}

The review protocol was preregistered in the PROSPERO database (CRD42017072280). This protocol adheres to the Preferred Reporting Items for Systematic Reviews and Meta-Analyses-Protocols 2015 guidelines for preparing protocols of systematic reviews ${ }^{23}$ (online supplementary material).

\section{STUDY DESIGN}

The Preferred Reporting Items for Systematic Reviews and Meta-Analyses (PRISMA) will guide the review.

\section{STUDY ELIGIBILITY}

Eligible studies will include:

- randomised and non-randomised controlled trials

- cross-sectional

- longitudinal studies

- case studies

- ethnographies and other types of qualitative research (eg, grounded theory, action research)

- systematic review and meta-analyses.

Eligible papers will address the question of 'who in the community are targeted in mHealth intervention?' Studies will be selected if mHealth interventions fit the definition of mHealth by Robert et al' : 'mobile computing, medical sensor, and communications technologies for health care' ${ }^{24}$ Furthermore, studies will need to be captured within one of the 12 common ICT application frameworks for maternal health proposed by Labrique et $a l .{ }^{25}$ These criteria are further discussed in the following section on study inclusion and exclusion.

\section{Inclusion/exclusion}

The participants, interventions, comparators and outcomes for this review are:

\section{Participants (P)}

All individuals or groups within communities (eg, husbands/spouses, mothers-in-law, family members, household members) (excluding health workers) who have been involved in a mHealth intervention geared at improving maternal health knowledge, behaviours and outcomes. Participants will be limited to those in LMIC as defined by the World Bank's classification scheme. ${ }^{26}$

\section{Interventions (I)}

mHealth interventions including SMS or text messages, mobile applications and phone calls which address maternal health issues, such as attendance at ANC visits, receipt and consumption of folic acid tablets, delivering with a skilled birth attendant or at a health institution, use of family-planning services, will be included. Health technologies will also include unidirectional or multidirectional messaging services (SMS or voice messages). Applications installed on patient smartphones/tablets or PDAs or those used by health 
workers, to promote maternal health-seeking behaviour in (pregnant) women or women of reproductive age, for which maternal health outcomes were measured. mHealth interventions that include women as direct end-users, alongside health workers or professionals for which maternal health outcomes were measured, will also be included.

In table 1 we present an overview of the 12 common mHealth and information communication technology applications based on Labrique $e t a l,{ }^{25}$ examples of mobile phone functions and our decision to include or exclude. For example, the first group of applications is for use in client education and behaviour-change communication. When these are used among participants of interest in the review, this category is applicable and thus included. Conversely, electronic health records are not applicable to our participants and thus such applications will be excluded.

\section{Comparators (C)}

Control groups may have received either the standard of care or other maternal interventions without an mHealth component.

\section{Outcomes (0)}

Four primary maternal health outcomes will be identified (a) knowledge-related outcomes, including knowledge of required of ANC visits, danger signs during pregnancy and delivery, appropriate contraceptive use; (b) attitudinal changes, such as increased willingness to attend ANC and motivation; (c) perceptions of recommended maternal health and family planning behaviours and quality of care; (d) change in maternal health-seeking behaviours or family planning practices, such as increased attendance at antenatal clinics, delivery at health facilities and utilisation of family-planning method. For our review, we have adopted an expanded definition of maternal health which includes family planning as a key component in reducing maternal mortality.

\section{Exclusion criteria}

Studies will be excluded if they focus on outcomes not directly related to maternal health as defined earlier. This includes studies on sexually transmitted infections such as chlamydia or HIV. Studies on specific subpopulations such as sex workers will also be excluded. Protocols for research studies or reviews will be excluded.

\section{Search criteria}

Searches will be conducted on articles published between 1999 and July 2017, to capture all articles since the emergence of mHealth technology in the health literature. ${ }^{27}$

We will search the following databases: PubMed, Web of Knowledge (Science Direct, EMBASE) and Cochrane Central Registers of Controlled Trials. Reference lists of included articles will also be reviewed for other relevant articles for inclusion. We will also search other databases for grey literature including 'mHealth Alliance' and 'mHealth Evidence'. ${ }^{28}$ Searches will be limited to English articles. Search terms will consist of Medical Subject Headings (MeSH), title/abstract (tiab) and text words (tw). Search terms will focus on mHealth, LMIC and maternal health. The proposed strategy can be found in table 2 .

\section{Selection of studies}

After the search strategy has been finalised and run on all search engines, they will be extracted. All extracted articles will be stored and managed in Mendeley reference manager. Duplicates will be identified and deleted using Mendeley duplicate identification tools. First-level inclusion: OI and I-OOA will conduct title and abstract screening of articles based on the inclusion and exclusion criteria. Second-level inclusion: full-text reading of all included articles by OI and I-OOA. SVB and MD will make the final decisions whenever the first two authors are unable to reach a consensus. Reasons for decisions taken at both levels of inclusion will be noted in an Excel sheet. The inclusion and exclusion processes will also be reported in a PRISMA flow chart. ${ }^{23}$

\section{Data extraction}

OI will develop a data-extraction form to be used throughout the review process. OI and I-OOA will pilot the form on a subset of 10 articles to assess the functionality and suitability of the form. Discussions will be held with the entire review team (OI, I-OOA, SVB, AB, JEWB and $\mathrm{MD}$ ) and adjustments made to the form before data extraction commences. Key information to be extracted can be found in table 3 .

\section{Data synthesis}

Extracted data will be summarised using narrative synthesis (NS) as described by Popay et al. ${ }^{29}$ NS primarily involves the usage of words and text to summarise review findings. NS has four key elements which we present below $^{29}$ :

1. Developing a theory of how the intervention works, why and for whom: there are no specific tools recommended for the design of a programme theory. The development of a programme theory will be guided by findings from the other three elements of an NS.

2. Developing a preliminary synthesis of findings of included studies: tabulation will be used to represent the quantitative and qualitative studies included in the review. This will include information on the study design, participant characteristics, intervention details, setting/context and outcome measures.

3. Exploring relationships in the data: conceptual/thematic models/groups will be developed across and within groups of similar articles. These groups would allow for the identification of similar findings.

4. Assessing the robustness of the synthesis: quality of the synthesis will be assessed through a critical reflection on the synthesis process. This will be presented in the Discussion section of the systematic review and will 
Table 1 Intervention inclusion and exclusion list based on 12 common applications and a visual framework (Labrique et al) ${ }^{25}$

\section{Common mHealth and ICT} applications

\begin{tabular}{|c|c|c|}
\hline $\begin{array}{l}\text { Client education and behaviour-change } \\
\text { communication }\end{array}$ & $\begin{array}{l}\text { SMS } \\
\text { MMS } \\
\text { IVR } \\
\text { - Voice communication/audio clips } \\
\text { - Video clips } \\
\text { Images }\end{array}$ & Included \\
\hline Sensors and point-of-care diagnostics & $\begin{array}{l}\text { Mobile phone camera } \\
\text { Tethered accessory sensors, devices } \\
\text { Built-in accelerometer } \\
\text { SMS } \\
\text { - Voice communication } \\
\text { Digital forms }\end{array}$ & $\begin{array}{l}\text { Included if community members/patients } \\
\text { participate in the diagnostic process. }\end{array}$ \\
\hline
\end{tabular}

$\begin{array}{lll}\text { Registries and vital events tracking } & \text { SMS } & \text { Included if community members/patients } \\ & \text { - Voice communication } & \text { are responsible for data collection. }\end{array}$

\begin{tabular}{|c|c|c|}
\hline Electronic health records & $\begin{array}{l}\text { SMS } \\
\text { Digital forms } \\
\text { Mobile web }\end{array}$ & Excluded \\
\hline
\end{tabular}

Electronic decision support (information, protocols, algorithms, checklists)

- Mobile web (WAP/GPRS)

- Stored information 'apps'

- IVR

$\begin{array}{lll}\text { Provider-to-provider communication } & \text { SMS } & \text { Excluded } \\ \text { (user groups, consultation) } & \text { MMS } \\ & & \text { Mobile phone camera }\end{array}$

Provider work planning and scheduling Interactive electronic client lists

Provider training and education

- SMS alerts

- Mobile phone calendar

\begin{tabular}{|c|c|c|}
\hline Human resource management & $\begin{array}{l}\text { MMS } \\
\text { - IVR } \\
\text { - Voice communication } \\
\text { - Audio or video clips, images } \\
\text { - Web-based performance dashboards } \\
\text { - GPS } \\
\text { - Voice communication } \\
\text { - SMS }\end{array}$ & Excluded \\
\hline Supply chain management & $\begin{array}{l}\text { Web-based supply dashboards } \\
\text { - GPS } \\
\text { - Digital forms } \\
\text { - SMS }\end{array}$ & Excluded \\
\hline Financial transactions and incentives & $\begin{array}{l}\text { Mobile money transfers and banking } \\
\text { services } \\
\text { Transfer of airtime minutes }\end{array}$ & $\begin{array}{l}\text { Included when patients/community } \\
\text { members directly receive incentives } \\
\text { as part of the mHealth intervention } \\
\text { and maternal and reproductive health } \\
\text { outcomes are reported. }\end{array}$ \\
\hline
\end{tabular}

GPRS, general packet radio service; GPS, global positioning service; ICT, information communication technology; IVR, interactive voice response; $\mathrm{mHealth}$, mobile health; MMS, multimedia messaging service; SMS, short message service; WAP, wireless application protocol. 
Table 2 PubMed search strategy, to be adapted for use in other database searches

\section{\#Column Searches}

\begin{tabular}{|c|c|}
\hline$\# 1$ & $\begin{array}{l}\text { User-Computer Interface[Mesh] OR multimedia[Mesh] OR cell phones[Mesh] OR computers, handheld[Mesh] } \\
\text { OR Mobile Applications[Mesh] OR mobile health[tiab] OR mhealth[tiab] OR m-health[tiab] OR ehealth[tiab] } \\
\text { OR e-health[tiab] OR digital health[tiab] OR smartphone[tiab] OR smartphones[tiab] OR phone[tiab] OR } \\
\text { phones[tiab] OR cellphone[tiab] OR cellphones[tiab] OR telephone[tiab] OR mobile application[tiab] OR mobile } \\
\text { applications[tiab] OR mobile technolog*[tiab] OR health technolog*[tiab] OR health application[tiab] OR health } \\
\text { applications[tiab] OR iPad[tiab] OR sms[tiab] OR mms[tiab] OR text messag*[tiab] OR USSD[tiab] OR pda[tiab] } \\
\text { OR laptop*[tiab] OR palmtop*[tiab] OR palm-top*[tiab] OR Personal Digital Assistant*[tiab] OR computer[tiab] OR } \\
\text { interactive voice response[tiab] OR multimedia[tiab] }\end{array}$ \\
\hline \# & \#1 AND \#2 AND \#3 \\
\hline
\end{tabular}

MeSH, Medical Subject Headings; tiab, title/abstract; tw, text words.

include reflection on the NS methodology, reviewers' assumptions and factors that could have influenced review findings.

Despite the linear presentation of the elements, the synthesis process is often iterative. A synthesis process flow chart will be included in the systematic review to display the process.

\section{Missing data}

When data is missing from articles and not publicly available, attempts will be made to contact study authors directly for the resource.

\section{Quality of evidence}

Quality of evidence will be assessed using method-appropriate tools. The Downs and Black ${ }^{30}$ checklist for quantitative healthcare studies will be used for all quantitative studies. This checklist was chosen for its multiple advantages, including its usefulness for both randomised controlled studies and non-randomised control studies and its being tailored for healthcare interventions. Quality appraisal for qualitative studies will be conducted using the guidelines proposed by Mays and Pope. ${ }^{31}$ These include questions about the worth and relevance of the work, clarity of research questions, appropriateness of the study design to the question, study context, sample and data collection, and analysis. If possible, overall quality of studies will be evaluated using the Grading of Recommendations Assessment, Development and Evaluation framework for quality of evidence. ${ }^{32}{ }^{33}$ Quality-appraisal tables and figures will be presented as additional files. 
Table 3 Components of data-extraction form

\section{Data to be extracted}

Article description

\section{Specific Items to be extracted}

Authors

Article title

Year of publication

Journal

Study setting

Study type

Study design

Region (sub-Saharan Africa, North Africa, Southeast Asia, etc)

Country

Setting (eg, rural/urban)

\begin{tabular}{|c|c|}
\hline Theoretical/conceptual framework & $\begin{array}{l}\text { Is there a theoretical or conceptual framework used? } \\
\text { If yes, what is it? } \\
\text { How do results and discussions relate to the theoretical or conceptual framework? }\end{array}$ \\
\hline Intervention description & $\begin{array}{l}\text { Type of mHealth tool use (mobile phone, smart phone, PDA, tablet) } \\
\text { Format of intervention (unidirectional or bidirectional messaging, voice messages, } \\
\text { application) } \\
\text { Description of intervention implementation }\end{array}$ \\
\hline Participant characteristics & $\begin{array}{l}\text { Target group (women, men, grandmothers, etc) } \\
\text { Ages } \\
\text { Educational level } \\
\text { Is a group described or an individual? } \\
\text { Geared towards pregnant women or pregnant women plus? } \\
\text { Couple? } \\
\text { Community/groups within community? } \\
\text { Pregnant or prepartum/post partum? } \\
\text { Intervention delivery }\end{array}$ \\
\hline Study measures and analysis & $\begin{array}{l}\text { Sampling and recruitment procedure } \\
\text { Data collection } \\
\text { Research tools } \\
\text { Analysis methods }\end{array}$ \\
\hline Outcomes measured & $\begin{array}{l}\text { Maternal or reproductive health knowledge } \\
\text { Maternal or reproductive health-seeking attitudes } \\
\text { Perceptions of maternal or reproductive healthcare } \\
\text { Maternal or reproductive health-seeking behaviours }\end{array}$ \\
\hline Results & Findings attributable to $\mathrm{mHealth}$ interventions \\
\hline Authors interpretations/conclusions & Quality of evidence \\
\hline Reviewers' comments & $\begin{array}{l}\text { Equity/sustainability effects } \\
\text { Implementation modalities } \\
\text { Implementation challenges/bottlenecks }\end{array}$ \\
\hline
\end{tabular}

mHealth, mobile health; PDA, personal digital assistant.

\section{ETHICS AND DISSEMINATION}

The sustained and increasing interest in mHealth over the last decades has led to an increase in mHealth interventions in reproductive health in LMIC. However, it is not clear what the role of targeting strategies is in the implementation of interventions (and the achievement of outcomes) and how these are related to reproductive health decision-making roles in different contexts. An understanding of this question is fundamental in future design and implementation of maternal and reproductive mHealth, and potentially other mHealth interventions in LMIC.

Acknowledgements The authors would like to thank Ralph de Vries, Vrije Universiteit Library, for his assistance in developing and refining the search strategy.
Collaborators Ralph de Vries.

Contributors $\mathrm{OI}, \mathrm{I}-\mathrm{OOA}, \mathrm{MD}$ and SVB conceived the review design and participated in refining the manuscript. $A B$ and JEWB contributed to refining the review design and OI drafted the manuscript. All authors read and approved the final manuscript.

Funding This work was supported by Erasmus Mundus Joint Doctorate Fellowship, Framework Partnership Agreement 2013-0039, Specific Grant Agreement 2014-0681.

Disclaimer The funder had no role in the decision to write and publish this protocol.

Competing interests None declared.

Patient consent Not required.

Provenance and peer review Not commissioned; externally peer reviewed.

Open Access This is an Open Access article distributed in accordance with the Creative Commons Attribution Non Commercial (CC BY-NC 4.0) license, which permits others to distribute, remix, adapt, build upon this work non-commercially, and license their derivative works on different terms, provided the original work is 
properly cited and the use is non-commercial. See: http://creativecommons.org/ licenses/by-nc/4.0/

(C) Article author(s) (or their employer(s) unless otherwise stated in the text of the article) 2018. All rights reserved. No commercial use is permitted unless otherwise expressly granted.

\section{REFERENCES}

1. Robert I, Swamy L, Pattichis Constantinos S. M-health:emerging mobile health systems. M-health emerg mob heal syst. US: Springer, 2006:623.

2. Labrique $A B$, Vasudevan $L$, Kochi $E$, et al. mHealth innovations as health system strengthening tools: 12 common applications and a visual framework. Global Health: Science and Practice 2013;1:160-71.

3. United Nations. The millennium development goals report. 2015 http://www.un.org/millenniumgoals/2015_MDG_Report/pdf/MDG 2015 rev (July 1).pdf (accessed 19 Jan 2017).

4. Lund S, Nielsen BB, Hemed M, et al. Mobile phones improve antenatal care attendance in Zanzibar: a cluster randomized controlled trial. BMC Pregnancy Childbirth 2014;14:29.

5. Castaño PM, Bynum JY, Andrés R, et al. Effect of daily text messages on oral contraceptive continuation. Obstetrics \& Gynecology 2012;119:14-20.

6. Chamberlain C, O'Mara-Eves A, Porter J, et al. Psychosocial interventions for supporting women to stop smoking in pregnancy. Cochrane Database Syst Rev 2017;2:CD001055.

7. Coleman J, Bohlin KC, Thorson A, et al. Effectiveness of an SMSbased maternal mHealth intervention to improve clinical outcomes of HIV-positive pregnant women. AIDS Care 2017;29:890-7.

8. Hou MY, Hurwitz S, Kavanagh E, et al. Using daily text-message reminders to improve adherence with oral contraceptives. Obstetrics \& Gynecology 2010;116:633-40.

9. Lau YK, Cassidy T, Hacking D, et al. Antenatal health promotion via short message service at a midwife obstetrics unit in south africa: a mixed methods study. BMC Pregnancy Childbirth 2014;14:284.

10. Homan FF, Hammond CS, Thompson EF, et al. Post-conflict transition and sustainability in Kosovo: establishing primary healthcare-based antenatal care. Prehosp Disaster Med 2010;25:28-33.

11. Khorshid MR, Afshari P, Abedi P. The effect of SMS messaging on the compliance with iron supplementation among pregnant women in Iran: a randomized controlled trial. $J$ Telemed Telecare 2014;20:201-6.

12. Lee $\mathrm{SH}$, Nurmatov UB, Nwaru $\mathrm{BI}$, et al. Effectiveness of mHealth interventions for maternal, newborn and child health in low- and middle-income countries: systematic review and meta-analysis. $J$ Glob Health 2016;6:10401.

13. Sondaal SF, Browne JL, Amoakoh-Coleman M, et al. Assessing the effect of mhealth interventions in improving maternal and neonatal care in low- and middle-income countries: a systematic review. PLoS One 2016:11:e0154664.

14. Tamrat T, Kachnowski S. Special delivery: an analysis of mHealth in maternal and newborn health programs and their outcomes around the world. Matern Child Health J 2012;16:1092-101.

15. Agarwal S, Rosenblum L, Goldschmidt T, et al. Mobile technology in support of frontline health workers a comprehensive overview of the landscape, knowledge gaps and future directions. https://dl. dropboxusercontent.com/u/5243748/mFHW Landscape_2016 Final. pdf (accessed 14 Mar 2017).

16. Agarwal S, Perry HB, Long LA, et al. Evidence on feasibility and effective use of mHealth strategies by frontline health workers in developing countries: systematic review. Trop Med Int Health 2015;20:1003-14.

17. Braun R, Catalani C, Wimbush J, et al. Community health workers and mobile technology: a systematic review of the literature. PLOS One 2013;8:e65772.

18. Cormick G, Kim NA, Rodgers A, et al. Interest of pregnant women in the use of SMS (short message service) text messages for the improvement of perinatal and postnatal care. Reprod Health 2012;9:9.

19. Chib A, van Velthoven MH, Car J. mHealth adoption in low-resource environments: a review of the use of mobile healthcare in developing countries. J Health Commun 2015;20:4-34.

20. Munyua S, Rotich G, Kimwele M. Factors affecting the adoption of mhealth in maternal health care in nakuru provincial general hospital. Int J Sci Technol Res Vol 2015;4:318-31.

21. Speizer IS, Whittle L, Carter M. Gender relations and reproductive decision making in Honduras. Int Fam Plan Perspect 2005;31:131-9.

22. Dudgeon MR, Inhorn MC. Men's influences on women's reproductive health: medical anthropological perspectives. Soc Sci Med 2004;59:1379-95.

23. Moher D, Shamseer L, Clarke M, et al. Preferred reporting items for systematic review and meta-analysis protocols (PRISMA-P) 2015 statement. Syst Rev 2015;4:1.

24. Grajales FJ, Sheps S, Ho K, et al. Social media: a review and tutorial of applications in medicine and health care. $J$ Med Internet Res 2014;16:e13.

25. Labrique $A B$, Vasudevan $L$, Kochi $E$, et al. mHealth innovations as health system strengthening tools: 12 common applications and a visual framework. Glob Health Sci Pract 2013;1:160-71.

26. World Bank Country and Lending Groups - World Bank Data Help Desk. https://datahelpdesk.worldbank.org/knowledgebase/articles/ 906519-world-bank-country-and-lending-groups (accessed 1 Aug 2017).

27. United Nations Foundation. mhealth for development the opportunity of mobile technology for healthcare in the developing world. Washington, D.C: United Nations Foundation, 2009. (accessed 26 Jul 2017).

28. mHealth Alliance. http://www.mhealthknowledge.org/resource-type/ mhealth-alliance (accessed 18 Aug 2017).

29. Popay J, Roberts H, Sowden A, et al. Guidance on the conduct of narrative synthesis in systematic reviews a product from the ESRC methods programme. England: Lancaster University, 2006. (accessed 18 Jul 2017).

30. Downs SH, Black N. The feasibility of creating a checklist for the assessment of the methodological quality both of randomised and non-randomised studies of health care interventions. J Epidemiol Community Health 1998;52:377-84.

31. Mays N, Pope C. Qualitative research in health care. Assessing quality in qualitative research. BMJ 2000;320:50-2.

32. Guyatt GH, Oxman AD, Vist GE, et al. GRADE: an emerging consensus on rating quality of evidence and strength of recommendations. BMJ 2008;336:924-6.

33. Guyatt $G$, Oxman AD, Akl EA, et al. GRADE guidelines: 1 . Introduction-GRADE evidence profiles and summary of findings tables. J Clin Epidemiol 2011;64:383-94. 